\title{
On some combinatorial relations for Tornheim's double series
}

\author{
by
}

\author{
Hirofumi Tsumura (Tokyo)
}

1. Introduction. Tornheim's double series $T(r, s, t)$ is defined by

$$
T(r, s, t)=\sum_{m, n=1}^{\infty} \frac{1}{m^{r} n^{s}(m+n)^{t}},
$$

where $r, s, t$ are nonnegative integers with $r+t>1, s+t>1$ and $r+s+t>2$, and was introduced in [5]. Tornheim showed that $T(r, s, N-r-s)$ is a polynomial in $\{\zeta(j) \mid 2 \leq j \leq N\}$ with rational coefficients when $N$ is odd and $N \geq 3$ (see [5, Theorem 7]). Recently Huard, Williams and Zhang Nan-Yue gave an explicit formula for $T(r, s, N-r-s)$ as a rational linear combination of the products $\zeta(2 j) \zeta(N-2 j)(0 \leq j \leq(N-3) / 2)$ when $N$ is odd, $N \geq 3$, and $r, s$ are nonnegative integers satisfying $1 \leq r+s \leq N-1$, $r \leq N-2$, and $s \leq N-2$ (see [2, Theorem 2]). On the other hand, it is an open problem to determine an explicit formula for $T(r, s, N-r-s)$ when $N$ is even. Indeed, only a few cases have been determined (see e.g. [2, p. 116]). In [4], Subbarao and Sitaramachandrarao gave an explicit formula for

$$
T(2 k, 2 p, 2 q)+T(2 k, 2 q, 2 p)+T(2 p, 2 q, 2 k)
$$

for $k, p, q \geq 1$. This can be viewed as a generalization of Mordell's result for $T(2 k, 2 k, 2 k)$ given in [3].

The purpose of this paper is to give some relations for $T(r, s, t)$ and their alternating analogues. Indeed, as a generalization of (1.2), we determine

$$
T(r, s, t)+(-1)^{r} T(r, t, s)+(-1)^{r+t} T(t, s, r),
$$

where $r, s \geq 0$ with $r+s \geq 2$ and $t \geq 2$ (see Theorem 1 ). In particular when $N=r+s+t$ is odd, we recover Tornheim's theorem mentioned above (see Remark after Theorem 1). When $N$ is even, for example, we give an explicit

2000 Mathematics Subject Classification: Primary 11M06; Secondary 30B99, 33E20, 40A05, 40B05. 
formula for

$$
T(2 k+1,2 p, 2 q+1)-T(2 k+1,2 q+1,2 p)+T(2 p, 2 q+1,2 k+1)
$$

(see Theorem 2). As an application, we give some relations for alternating analogues of $T(r, s, t)$ (see Proposition 3). In particular, we give an explicit formula for

$$
\sum_{m, n=1}^{\infty} \frac{(-1)^{m+n}}{m^{2 k+1} n^{2 k+1}(m+n)^{2 k+1}}
$$

for $k \in \mathbb{N} \cup\{0\}$ (see Corollary 3). The problem of evaluating (1.4) was posed in $[4, \S 5]$.

The author would like to express his sincere gratitude to the referee for his valuable comments.

2. Preliminaries. Let $\mathbb{N}$ be the set of natural numbers, $\mathbb{Z}$ the ring of rational integers, and $\mathbb{R}$ the field of real numbers. Throughout this paper we fix $\delta \in \mathbb{R}$ with $\delta>0$. For $u \in \mathbb{R}$ with $1 \leq u \leq 1+\delta$ and $s \in \mathbb{Z}$, define

$$
\phi(s ; u):=\sum_{m=1}^{\infty} \frac{(-u)^{-m}}{m^{s}} .
$$

If $u>1$ then $\phi(s ; u)$ is convergent for any $s \in \mathbb{Z}$. In the case when $u=1$, let $\phi(s):=\phi(s ; 1)=\left(2^{1-s}-1\right) \zeta(s)$. Corresponding to $\phi(s ; u)$, we define a set $\left\{\varepsilon_{m}(u)\right\}$ of numbers by

$$
G(x ; u):=\frac{(1+u) e^{x}}{e^{x}+u}=\sum_{m=0}^{\infty} \varepsilon_{m}(u) \frac{x^{m}}{m !} .
$$

When $u=1$, it is well known that

$$
G(x ; 1)=\frac{2 e^{x}}{e^{x}+1}=\sum_{m=0}^{\infty} E_{m}(1) \frac{x^{m}}{m !},
$$

where $E_{m}(x)$ is the $m$ th Euler polynomial (see e.g. [1]). Hence

$$
\varepsilon_{2 j}(1)=E_{2 j}(1)=0 \quad(j \in \mathbb{N}),
$$

and if $u \in[1,1+\delta]$, then

$$
\liminf _{m \rightarrow \infty}\left(\frac{\left|\varepsilon_{m}(u)\right|}{m !}\right)^{-1 / m} \geq \pi .
$$

From (2.1) and (2.2), we have 
Lemma 1. $\phi(-k ; u)=-\varepsilon_{k}(u) /(1+u)$ for $k \in \mathbb{N} \cup\{0\}$ and $u \in(1,1+\delta]$. By Lemma 1, we have

$$
\begin{aligned}
I_{2 k}(\theta ; u) & :=\sum_{m=1}^{\infty} \frac{(-u)^{-m} \cos (m \theta)}{m^{2 k}}-\sum_{j=0}^{k} \phi(2 k-2 j ; u) \frac{(i \theta)^{2 j}}{(2 j) !} \\
& =-\frac{1}{1+u} \sum_{m=1}^{\infty} \varepsilon_{2 m}(u) \frac{(i \theta)^{2 m+2 k}}{(2 m+2 k) !}
\end{aligned}
$$

and

$$
\begin{aligned}
J_{2 k+1}(\theta ; u) & :=i \sum_{m=1}^{\infty} \frac{(-u)^{-m} \sin (m \theta)}{m^{2 k+1}}-\sum_{j=0}^{k} \phi(2 k-2 j ; u) \frac{(i \theta)^{2 j+1}}{(2 j+1) !} \\
& =-\frac{1}{1+u} \sum_{m=1}^{\infty} \varepsilon_{2 m}(u) \frac{(i \theta)^{2 m+2 k+1}}{(2 m+2 k+1) !}
\end{aligned}
$$

for $k, l \in \mathbb{N} \cup\{0\}$. Combining (2.3) and (2.4), we obtain

Lemma 2. Suppose $k \in \mathbb{N} \cup\{0\}$ and $\theta \in(-\pi, \pi)$. Then $I_{2 k}(\theta ; u)$ and $J_{2 k+1}(\theta ; u)$ are uniformly convergent with respect to $u \in(1,1+\delta]$, and satisfy $I_{2 k}(\theta ; u) \rightarrow 0$ and $J_{2 k+1}(\theta ; u) \rightarrow 0$ as $u \rightarrow 1$.

For $u \in(1,1+\delta], s \in \mathbb{Z}$ and $k, l \in \mathbb{N} \cup\{0\}$, define

$$
\begin{aligned}
& R(k, s, l ; u):=\sum_{m, n=1}^{\infty} \frac{(-u)^{-(2 m+n)}}{m^{k} n^{s}(m+n)^{l}}, \\
& S(k, l, s ; u):=\sum_{m, n=1}^{\infty} \frac{(-u)^{-(m+n)}}{m^{k} n^{l}(m+n)^{s}} .
\end{aligned}
$$

Lemma 3. Suppose $k, l \in \mathbb{N} \cup\{0\}, \theta \in(-\pi, \pi)$ and $u \in(1,1+\delta]$. Then

$$
\begin{aligned}
& \sum_{m=1}^{\infty} \frac{(-u)^{-m} e^{i m \theta}}{m^{k}} \cdot \sum_{n=1}^{\infty} \frac{(-u)^{-n} e^{i n \theta}}{n^{l}}=\sum_{m=0}^{\infty} S(k, l,-m ; u) \frac{(i \theta)^{m}}{m !} \\
& \sum_{m=1}^{\infty} \frac{(-u)^{-m} e^{i m \theta}}{m^{k}} \cdot \sum_{n=1}^{\infty} \frac{(-u)^{-n} e^{-i n \theta}}{n^{l}} \\
& =\sum_{m=0}^{\infty}\left\{R(l,-m, k ; u)+(-1)^{m} R(k,-m, l ; u)\right\} \frac{(i \theta)^{m}}{m !}+\sum_{m=1}^{\infty} \frac{u^{-2 m}}{m^{k+l}}
\end{aligned}
$$


Proof. By (2.8), we immediately obtain (2.9). The left-hand side of (2.10) is equal to

$$
\begin{gathered}
\sum_{\substack{m, n=1 \\
m>n}}^{\infty} \frac{(-u)^{-(m+n)} e^{i(m-n) \theta}}{m^{k} n^{l}}+\sum_{m=1}^{\infty} \frac{u^{-2 m}}{m^{k+l}}+\sum_{\substack{m, n=1 \\
m<n}}^{\infty} \frac{(-u)^{-(m+n)} e^{-i(n-m) \theta}}{m^{k} n^{l}} \\
=\sum_{n, j=1}^{\infty} \frac{(-u)^{-(2 n+j)} e^{i j \theta}}{n^{l}(n+j)^{k}}+\sum_{m=1}^{\infty} \frac{u^{-2 m}}{m^{k+l}}+\sum_{m, j=1}^{\infty} \frac{(-u)^{-(2 m+j)} e^{-i j \theta}}{m^{k}(m+j)^{l}} .
\end{gathered}
$$

Hence, by (2.7), we obtain (2.10).

Lemma 4. For $k, l \in \mathbb{N} \cup\{0\}, \theta \in(-\pi, \pi)$ and $u \in(1,1+\delta]$,

$$
I_{2 k}(\theta ; u)\left(\sum_{n=1}^{\infty} \frac{(-u)^{-n} e^{i n \theta}}{n^{l}}\right)
$$

$$
\begin{aligned}
= & \sum_{m=0}^{\infty} \frac{1}{2}\left\{S(2 k, l,-m ; u)+R(2 k,-m, l ; u)+(-1)^{m} R(l,-m, 2 k ; u)\right\} \frac{(i \theta)^{m}}{m !} \\
& -\sum_{m=0}^{\infty} \sum_{j=0}^{k}\left(\begin{array}{l}
m \\
2 j
\end{array}\right) \phi(2 k-2 j ; u) \phi(l+2 j-m ; u) \frac{(i \theta)^{m}}{m !}+\frac{1}{2} \sum_{m=1}^{\infty} \frac{u^{-2 m}}{m^{2 k+l}},
\end{aligned}
$$

$$
\begin{aligned}
& J_{2 k+1}(\theta ; u)\left(\sum_{n=1}^{\infty} \frac{(-u)^{-n} e^{i n \theta}}{n^{l}}\right) \\
& =\sum_{m=0}^{\infty} \frac{1}{2}\{S(2 k+1, l,-m ; u)-R(2 k+1,-m, l ; u) \\
& \left.\quad-(-1)^{m} R(l,-m, 2 k+1 ; u)\right\} \frac{(i \theta)^{m}}{m !} \\
& \quad-\sum_{m=0}^{\infty} \sum_{j=0}^{k}\left(\begin{array}{c}
m \\
2 j+1
\end{array}\right) \phi(2 k-2 j ; u) \phi(l+2 j+1-m ; u) \frac{(i \theta)^{m}}{m !} \\
& \quad-\frac{1}{2} \sum_{m=1}^{\infty} \frac{u^{-2 m}}{m^{2 k+1+l}} .
\end{aligned}
$$

Proof. By the binomial theorem, we have

$$
\begin{aligned}
\left(\sum_{j=0}^{k} \phi(2 k-2 j ; u) \frac{(i \theta)^{2 j}}{(2 j) !}\right)\left(\sum_{n=1}^{\infty} \frac{(-u)^{-n} e^{i n \theta}}{n^{l}}\right) \\
=\left(\sum_{j=0}^{k} \phi(2 k-2 j ; u) \frac{(i \theta)^{2 j}}{(2 j) !}\right)\left(\sum_{m=0}^{\infty} \phi(l-m ; u) \frac{(i \theta)^{m}}{m !}\right) \\
=\sum_{N=0}^{\infty} \sum_{j=0}^{k}\left(\begin{array}{l}
N \\
2 j
\end{array}\right) \phi(2 k-2 j ; u) \phi(l+2 j-N ; u) \frac{(i \theta)^{N}}{N !}
\end{aligned}
$$


and

$$
\begin{gathered}
\left(\sum_{j=0}^{k} \phi(2 k-2 j ; u) \frac{(i \theta)^{2 j+1}}{(2 j+1) !}\right)\left(\sum_{n=1}^{\infty} \frac{(-u)^{-n} e^{i n \theta}}{n^{l}}\right) \\
=\sum_{N=0}^{\infty} \sum_{j=0}^{k}\left(\begin{array}{c}
N \\
2 j+1
\end{array}\right) \phi(2 k-2 j ; u) \phi(l+2 j+1-N ; u) \frac{(i \theta)^{N}}{N !} .
\end{gathered}
$$

By Lemma 3, we immediately obtain (2.11) and (2.12).

For $k, l \in \mathbb{N} \cup\{0\}, u \in(1,1+\delta]$ and $r \in \mathbb{Z}$, we define

$$
\begin{aligned}
& A(r ; 2 k, l ; u) \\
& :=\frac{1}{2}\left\{S(2 k, l, r ; u)+R(2 k, r, l ; u)+(-1)^{r} R(l, r, 2 k ; u)\right\} \\
& -\sum_{j=0}^{k}\left(\begin{array}{c}
r+2 j-1 \\
2 j
\end{array}\right) \phi(2 k-2 j ; u) \phi(l+2 j+r ; u)
\end{aligned}
$$

and

$$
\begin{aligned}
& A(r ; 2 k+1, l ; u) \\
:= & \frac{1}{2}\left\{S(2 k+1, l, r ; u)-R(2 k+1, r, l ; u)-(-1)^{r} R(l, r, 2 k+1 ; u)\right\} \\
& +\sum_{j=0}^{k}\left(\begin{array}{c}
r+2 j \\
2 j+1
\end{array}\right) \phi(2 k-2 j ; u) \phi(l+2 j+1+r ; u) .
\end{aligned}
$$

Lemma 5. Suppose $p, q \in \mathbb{N} \cup\{0\}$ with $p+q \geq 2$. Then

$$
\begin{gathered}
\liminf _{m \rightarrow \infty}\left(\frac{A(-m ; p, q ; u)}{m !}\right)^{-1 / m} \geq \pi \quad(u \in(1,1+\delta]), \\
\lim _{u \rightarrow 1} A(-m ; p, q ; u)=0 \quad(m \in \mathbb{N}), \\
\lim _{u \rightarrow 1} A(0 ; p, q ; u)=\frac{(-1)^{p+1}}{2} \zeta(p+q) .
\end{gathered}
$$

Proof. It follows from (2.4) and Lemma 2 that (2.11) and (2.12) are uniformly convergent with respect to $u \in(1,1+\delta]$ when $k, l \in \mathbb{N} \cup\{0\}$ with $2 k+l \geq 2$ and $\theta \in(-\pi, \pi)$ and that these tend to 0 as $u \rightarrow 1$. Hence each coefficient of $(i \theta)^{\mathrm{m}} / \mathrm{m}$ ! on the right-hand side of (2.11) and (2.12) tends to 0 as $u \rightarrow 1$. Then it follows from the well-known relation

$$
\left(\begin{array}{c}
-x \\
y
\end{array}\right)=(-1)^{y}\left(\begin{array}{c}
x+y-1 \\
y
\end{array}\right)
$$

that (2.15)-(2.17) hold. 
3. Relations for $T(r, s, t)$. We begin by proving the following combinatorial relations.

Lemma 6. For $c, s \in \mathbb{N} \cup\{0\}$,

$$
\begin{aligned}
\sum_{\nu=0}^{c} \frac{(-\theta)^{\nu}}{\nu !}\left(\begin{array}{c}
s+c-\nu \\
c-\nu
\end{array}\right) & \frac{\cos ^{(\nu)}(x \theta)}{x^{s+1+c-\nu}} \\
& =\sum_{N=0}^{\infty}\left(\begin{array}{c}
s+c-2 N \\
c
\end{array}\right) \frac{1}{x^{s+1+c-2 N}} \cdot \frac{(i \theta)^{2 N}}{(2 N) !}
\end{aligned}
$$

where $\cos ^{(\nu)}(\theta)$ is the $\nu$ th derivative of $\cos \theta$.

Proof. Let $f(x ; s, \theta)=\cos (x \theta) x^{-s-1}$. In order to prove (3.1), we have only to calculate the $c$ th derivative of $f(x ; s, \theta)$ with respect to $x$ as follows:

$$
\frac{d^{c}}{d x^{c}} f(x ; s, \theta)=\sum_{\nu=0}^{c}\left(\begin{array}{l}
c \\
\nu
\end{array}\right)\{\cos (x \theta)\}^{(\nu)} \cdot\left\{x^{-s-1}\right\}^{(c-\nu)}
$$

and

$$
\frac{d^{c}}{d x^{c}} f(x ; s, \theta)=\frac{d^{c}}{d x^{c}}\left\{\sum_{N=0}^{\infty} \frac{(i \theta)^{2 N}}{(2 N) !} x^{2 N-s-1}\right\},
$$

by using the laws of differentiation and the Maclaurin expansion of $\cos \theta$.

By applying this lemma, we obtain

$$
\begin{aligned}
\sum_{\nu=0}^{c} \frac{(-\theta)^{\nu}}{\nu !}\left(\begin{array}{c}
s+c-\nu \\
c-\nu
\end{array}\right) \sum_{m=1}^{\infty} \frac{(-u)^{-m} \cos ^{(\nu)}(m \theta)}{m^{s+1+c+l-\nu}} \\
=\sum_{N=0}^{\infty}\left(\begin{array}{c}
s+c-2 N \\
c
\end{array}\right) \sum_{m=1}^{\infty} \frac{(-u)^{-m}}{m^{s+1+c+l-2 N}} \cdot \frac{(i \theta)^{2 N}}{(2 N) !}
\end{aligned}
$$

for $c, l, s \in \mathbb{N} \cup\{0\}$. Hence we can prove

Lemma 7. Suppose $k, l \in \mathbb{N} \cup\{0\}, d \in \mathbb{N}, \theta \in(-\pi, \pi)$ and $u \in(1,1+\delta]$. Then

$$
\begin{array}{r}
\frac{1}{2} \sum_{m, n=1}^{\infty}\left\{\frac{(-u)^{-(m+n)} \cos ((m+n) \theta)}{m^{2 k} n^{l}(m+n)^{d}}+\frac{(-u)^{-(2 m+n)} \cos (n \theta)}{m^{2 k} n^{d}(m+n)^{l}}\right. \\
\left.+(-1)^{d} \frac{(-u)^{-(2 m+n)} \cos (n \theta)}{m^{l} n^{d}(m+n)^{2 k}}\right\}
\end{array}
$$




$$
\begin{aligned}
& -\sum_{j=0}^{k} \phi(2 k-2 j ; u) \sum_{\nu=0}^{2 j} \frac{(-\theta)^{\nu}}{\nu !}\left(\begin{array}{c}
d-1+2 j-\nu \\
2 j-\nu
\end{array}\right) \\
& \times \sum_{m=1}^{\infty} \frac{(-u)^{-m} \cos ^{(\nu)}(m \theta)}{m^{d+2 j+l-\nu}} \\
& =\sum_{N=0}^{\infty} A(d-2 N ; 2 k, l ; u) \frac{(i \theta)^{2 N}}{(2 N) !}, \\
& \frac{1}{2} \sum_{m, n=1}^{\infty}\left\{\frac{(-u)^{-(m+n)} \cos ((m+n) \theta)}{m^{2 k+1} n^{l}(m+n)^{d}}-\frac{(-u)^{-(2 m+n)} \cos (n \theta)}{m^{2 k+1} n^{d}(m+n)^{l}}\right. \\
& \left.-(-1)^{d} \frac{(-u)^{-(2 m+n)} \cos (n \theta)}{m^{l} n^{d}(m+n)^{2 k+1}}\right\} \\
& +\sum_{j=0}^{k} \phi(2 k-2 j ; u) \sum_{\nu=0}^{2 j+1} \frac{(-\theta)^{\nu}}{\nu !}\left(\begin{array}{l}
d+2 j-\nu \\
2 j+1-\nu
\end{array}\right) \\
& \times \sum_{m=1}^{\infty} \frac{(-u)^{-m} \cos ^{(\nu)}(m \theta)}{m^{d+2 j+1+l-\nu}} \\
& =\sum_{N=0}^{\infty} A(d-2 N ; 2 k+1, l ; u) \frac{(i \theta)^{2 N}}{(2 N) !} .
\end{aligned}
$$

In particular when $d \geq 2$, (3.3) and (3.4) hold for $\theta \in[-\pi, \pi]$.

Proof. Suppose $k, l \in \mathbb{N} \cup\{0\}, d \in \mathbb{N}$ and $\theta \in(-\pi, \pi)$. By (2.7) and (2.8), we have

$$
\sum_{m, n=1}^{\infty} \frac{(-u)^{-(2 m+n)} \cos (n \theta)}{m^{p} n^{q}(m+n)^{r}}=\sum_{m=0}^{\infty} R(p, q-2 m, r ; u) \frac{(i \theta)^{2 m}}{(2 m) !}
$$

and

$$
\sum_{m, n=1}^{\infty} \frac{(-u)^{-(m+n)} \cos ((m+n) \theta)}{m^{p} n^{q}(m+n)^{r}}=\sum_{m=0}^{\infty} S(p, q, r-2 m ; u) \frac{(i \theta)^{2 m}}{(2 m) !} .
$$

On the other hand, by applying (3.2) in the case $(c, s)=(2 j, d-1)$, we have

$$
\begin{aligned}
\sum_{\nu=0}^{2 j} \frac{(-\theta)^{\nu}}{\nu !}\left(\begin{array}{c}
d-1 \\
2 j-\nu
\end{array}\right) \sum_{m=1}^{\infty} \frac{(-u)^{-m} \cos ^{(\nu)}(m \theta)}{m^{d+2 j+l-\nu}} \\
=\sum_{N=0}^{\infty}\left(\begin{array}{c}
2 N-d \\
2 j
\end{array}\right) \phi(l+2 j+d-2 N ; u) \frac{(i \theta)^{2 N}}{(2 N) !} .
\end{aligned}
$$

Combining (2.13) and (3.5)-(3.7), we obtain the proof of (3.3). In particular 
when $d \geq 2$, it follows from (2.15) that (3.3) holds for $\theta \in[-\pi, \pi]$. In the same way as above, we can verify (3.4).

From (2.13), (2.14) and (2.17), we can define

$$
A(r ; p, q):=\lim _{u \rightarrow 1} A(r ; p, q ; u)
$$

for $p, q, r \in \mathbb{N} \cup\{0\}$ with $p+q \geq 2$. With this notation, we obtain

Proposition 1. Suppose $k, l \in \mathbb{N} \cup\{0\}, d \in \mathbb{N}$ with $d \geq 2$ and $\theta \in$ $[-\pi, \pi]$. When $2 k+l \geq 2$,

$$
\begin{aligned}
\frac{1}{2} \sum_{m, n=1}^{\infty}\left\{\begin{array}{c}
\frac{(-1)^{m+n} \cos ((m+n) \theta)}{m^{2 k} n^{l}(m+n)^{d}}+\frac{(-1)^{n} \cos (n \theta)}{m^{2 k} n^{d}(m+n)^{l}} \\
+\frac{(-1)^{d+n} \cos (n \theta)}{m^{l} n^{d}(m+n)^{2 k}}
\end{array}\right\} \\
\quad-\sum_{j=0}^{k} \phi(2 k-2 j) \sum_{\nu=0}^{2 j} \frac{(-\theta)^{\nu}}{\nu !}\left(\begin{array}{c}
d-1+2 j-\nu \\
2 j-\nu
\end{array}\right) \\
\quad \times \sum_{m=1}^{\infty} \frac{(-1)^{m} \cos ^{(\nu)}(m \theta)}{m^{d+2 j+l-\nu}} \\
=\sum_{N=0}^{[d / 2]} A(d-2 N ; 2 k, l) \frac{(i \theta)^{2 N}}{(2 N) !} .
\end{aligned}
$$

When $2 k+l \geq 1$,

$$
\begin{aligned}
\frac{1}{2} \sum_{m, n=1}^{\infty}\left\{\frac{(-1)^{m+n} \cos ((m+n) \theta)}{m^{2 k+1} n^{l}(m+n)^{d}}-\frac{(-1)^{n} \cos (n \theta)}{m^{2 k+1} n^{d}(m+n)^{l}}\right. & \left.-\frac{(-1)^{d+n} \cos (n \theta)}{m^{l} n^{d}(m+n)^{2 k+1}}\right\} \\
& +\sum_{j=0}^{k} \phi(2 k-2 j) \sum_{\nu=0}^{2 j+1} \frac{(-\theta)^{\nu}}{\nu !}\left(\begin{array}{l}
d+2 j-\nu \\
2 j+1-\nu
\end{array}\right) \\
& \times \sum_{m=1}^{\infty} \frac{(-1)^{m} \cos ^{(\nu)}(m \theta)}{m^{d+2 j+1+l-\nu}} \\
= & \sum_{N=0}^{[d / 2]} A(d-2 N ; 2 k+1, l) \frac{(i \theta)^{2 N}}{(2 N) !}
\end{aligned}
$$

Proof. By (2.15), we see that both sides of (3.3) and (3.4) are uniformly convergent with respect to $u \in(1,1+\delta]$ for $\theta \in[-\pi, \pi]$ when $d \geq 2$. Hence, 
by letting $u \rightarrow 1$ on both sides of (3.3) and (3.4), and by using (2.16), (2.17) and (3.8), we obtain the proofs of (3.9) and (3.10).

By applying Proposition 1 in the case $\theta=\pi$, we have the following relations for $T(r, s, t)$.

Corollary 1. Suppose $k, l \in \mathbb{N} \cup\{0\}$ and $d \in \mathbb{N}$ with $d \geq 2$. When $2 k+l \geq 2$,

$$
\begin{aligned}
& \frac{1}{2}\left\{T(2 k, l, d)+T(2 k, d, l)+(-1)^{d} T(l, d, 2 k)\right\} \\
& -\sum_{j=0}^{k} \phi(2 k-2 j) \sum_{\mu=0}^{j} \frac{(i \pi)^{2 \mu}}{(2 \mu) !}\left(\begin{array}{c}
d-1+2 j-2 \mu \\
2 j-2 \mu
\end{array}\right) \zeta(d+2 j+l-2 \mu) \\
= & \sum_{N=0}^{[d / 2]} A(d-2 N ; 2 k, l) \frac{(i \pi)^{2 N}}{(2 N) !}
\end{aligned}
$$

When $2 k+l \geq 1$,

$$
\begin{aligned}
& \frac{1}{2}\left\{T(2 k+1, l, d)-T(2 k+1, d, l)-(-1)^{d} T(l, d, 2 k+1)\right\} \\
& +\sum_{j=0}^{k} \phi(2 k-2 j) \sum_{\mu=0}^{j} \frac{(i \pi)^{2 \mu}}{(2 \mu) !}\left(\begin{array}{c}
d+2 j-2 \mu \\
2 j+1-2 \mu
\end{array}\right) \zeta(d+2 j+1+l-2 \mu) \\
= & \sum_{N=0}^{[d / 2]} A(d-2 N ; 2 k+1, l) \frac{(i \pi)^{2 N}}{(2 N) !} .
\end{aligned}
$$

Proposition 2. Suppose $k, l \in \mathbb{N} \cup\{0\}, d \in \mathbb{N}$ with $d \geq 3$ and $\theta \in$ $[-\pi, \pi]$. When $2 k+l \geq 2$,

$$
\begin{aligned}
& -\frac{1}{2} \sum_{m, n=1}^{\infty}\left\{\frac{(-1)^{m+n} \sin ((m+n) \theta)}{m^{2 k} n^{l}(m+n)^{d-1}}+\frac{(-1)^{n} \sin (n \theta)}{m^{2 k} n^{d-1}(m+n)^{l}}\right. \\
& \left.+\frac{(-1)^{d+n} \sin (n \theta)}{m^{l} n^{d-1}(m+n)^{2 k}}\right\} \\
& -\sum_{j=0}^{k} \phi(2 k-2 j) \sum_{\nu=0}^{2 j} \frac{(-\theta)^{\nu}}{\nu !}\left(\begin{array}{c}
d-2+2 j-\nu \\
2 j-\nu
\end{array}\right) \\
& \times \sum_{m=1}^{\infty} \frac{(-1)^{m} \cos ^{(\nu+1)}(m \theta)}{m^{d+2 j+l-\nu-1}} \\
& =\sum_{N=1}^{[d / 2]} A(d-2 N ; 2 k, l) \frac{(-1)^{N} \theta^{2 N-1}}{(2 N-1) !} .
\end{aligned}
$$


When $2 k+l \geq 1$,

$$
\begin{aligned}
& -\frac{1}{2} \sum_{m, n=1}^{\infty}\left\{\frac{(-1)^{m+n} \sin ((m+n) \theta)}{m^{2 k+1} n^{l}(m+n)^{d-1}}-\frac{(-1)^{n} \sin (n \theta)}{m^{2 k+1} n^{d-1}(m+n)^{l}}\right. \\
& \left.\quad-\frac{(-1)^{d+n} \sin (n \theta)}{m^{l} n^{d-1}(m+n)^{2 k+1}}\right\} \\
& \quad+\sum_{j=0}^{k} \phi(2 k-2 j) \sum_{\nu=0}^{2 j+1} \frac{(-\theta)^{\nu}}{\nu !}\left(\begin{array}{c}
d-1+2 j-\nu \\
2 j+1-\nu
\end{array}\right) \\
& \quad \times \sum_{m=1}^{\infty} \frac{(-1)^{m} \cos ^{(\nu+1)}(m \theta)}{m^{d+2 j+l-\nu}} \\
& =\sum_{N=1}^{[d / 2]} A(d-2 N ; 2 k+1, l) \frac{(-1)^{N} \theta^{2 N-1}}{(2 N-1) !} .
\end{aligned}
$$

Proof. We differentiate both sides of (3.3) and (3.4) with respect to $\theta$. For example, we can easily verify that

$$
\begin{aligned}
\frac{d}{d \theta}\left(\sum_{\nu=0}^{2 j} \frac{(-\theta)^{\nu}}{\nu !}\left(\begin{array}{c}
d-1+2 j-\nu \\
2 j-\nu
\end{array}\right) \sum_{m=1}^{\infty} \frac{(-u)^{-m} \cos ^{(\nu)}(m \theta)}{m^{d+2 j+l-\nu}}\right) \\
=\sum_{\nu=0}^{2 j} \frac{(-\theta)^{\nu}}{\nu !}\left(\begin{array}{c}
d-2+2 j-\nu \\
2 j-\nu
\end{array}\right) \sum_{m=1}^{\infty} \frac{(-u)^{-m} \cos ^{(\nu+1)}(m \theta)}{m^{d+2 j+l-\nu-1}},
\end{aligned}
$$

by using the well-known relation

$$
-\left(\begin{array}{l}
x-1 \\
y-1
\end{array}\right)+\left(\begin{array}{l}
x \\
y
\end{array}\right)=\left(\begin{array}{c}
x-1 \\
y
\end{array}\right)
$$

Since $d \geq 3$, both sides of the resulting equations are uniformly convergent with respect to $u \in(1,1+\delta]$ for $\theta \in[-\pi, \pi]$. By using the same method as in the proof of Proposition 1, we can prove (3.13) and (3.14).

By applying Proposition 2 in the case $\theta=\pi$, we have the following relations.

Corollary 2. Suppose $k, l \in \mathbb{N} \cup\{0\}$ and $d \in \mathbb{N}$ with $d \geq 3$. When $2 k+l \geq 2$,

$$
\begin{array}{r}
\sum_{j=0}^{k} \phi(2 k-2 j) \sum_{\mu=0}^{j-1} \frac{(i \pi)^{2 \mu}}{(2 \mu+1) !}\left(\begin{array}{c}
d-3+2 j-2 \mu \\
2 j-2 \mu-1
\end{array}\right) \zeta(d-2+2 j+l-2 \mu) \\
=\sum_{\nu=0}^{[d / 2]-1} A(d-2-2 \nu ; 2 k, l) \frac{(i \pi)^{2 \nu}}{(2 \nu+1) !}
\end{array}
$$


When $2 k+l \geq 1$,

$$
\begin{array}{r}
\sum_{j=0}^{k} \phi(2 k-2 j) \sum_{\mu=0}^{j} \frac{(i \pi)^{2 \mu}}{(2 \mu+1) !}\left(\begin{array}{c}
d-2+2 j-2 \mu \\
2 j-2 \mu
\end{array}\right) \zeta(d-1+2 j+l-2 \mu) \\
=-\sum_{\nu=0}^{[d / 2]-1} A(d-2-2 \nu ; 2 k+1, l) \frac{(i \pi)^{2 \nu}}{(2 \nu+1) !}
\end{array}
$$

It follows from Corollary 2 and $(2.17)$ that $A(p ; 2 k, l)$ and $A(p ; 2 k+1, l)$ are inductively determined as polynomials in $\zeta(j)$ with rational coefficients for $p \in \mathbb{N} \cup\{0\}$. Hence, by Corollary 1, we obtain

Theorem 1. Suppose $r, s \in \mathbb{N} \cup\{0\}$ with $r+s \geq 2$ and $t \in \mathbb{N}$ with $t \geq 2$. Then

$$
T(r, s, t)+(-1)^{r} T(r, t, s)+(-1)^{r+t} T(t, s, r)
$$

is a polynomial in $\{\zeta(j) \mid 2 \leq j \leq r+s+t\}$ with rational coefficients.

Remark. By applying Theorem 1 in the case when $r+s+t$ is odd, we immediately obtain Tornheim's theorem mentioned in Section 1. In particular, an explicit formula for (1.2) can be deduced from Theorem 1. But this is more complicated than in [4, Theorem 4.1].

4. Explicit formulas. In this section, we give some explicit formulas for $T(r, s, t)$ and their alternating series. We need the following elementary lemma which can be proved by formal calculation.

LEMma 8. Suppose $\left\{P_{m}\right\}$ and $\left\{Q_{m}\right\}$ are sequences which satisfy

$$
\sum_{\nu=0}^{m} P_{m-\nu} \frac{(i \pi)^{2 \nu}}{(2 \nu+1) !}=Q_{m} \quad \text { for any } m \in \mathbb{N} \cup\{0\} \text {. }
$$

Then

$$
P_{m}=-2 \sum_{\nu=0}^{m} \phi(2 m-2 \nu) Q_{\nu} \quad \text { for any } m \in \mathbb{N} \cup\{0\} .
$$

Proof. By the assumption, we have

$$
\begin{aligned}
\left(\sum_{m=0}^{\infty} P_{m} x^{2 m}\right) \frac{e^{i \pi x}-e^{-i \pi x}}{2} & =\sum_{m=0}^{\infty}\left\{\sum_{\nu=0}^{m} P_{m-\nu} \frac{(i \pi)^{2 \nu+1}}{(2 \nu+1) !}\right\} x^{2 m+1} \\
& =(i \pi x) \sum_{m=0}^{\infty} Q_{m} x^{2 m}
\end{aligned}
$$


Recalling the definition of the Bernoulli numbers (see e.g. [1, Chap. 1]), we can verify that

$$
\frac{(2 i \pi x) e^{i \pi x}}{e^{2 i \pi x}-1}=\sum_{n=0}^{\infty}\left(2-2^{2 n}\right) B_{2 n} \frac{(i \pi x)^{2 n}}{(2 n) !}=\sum_{n=0}^{\infty}\left(2-2^{2-2 n}\right) \zeta(2 n) x^{2 n} .
$$

Since $\phi(s)=\left(2^{1-s}-1\right) \zeta(s)$, we have

$$
\sum_{m=0}^{\infty} P_{m} x^{2 m}=\left(\sum_{k=0}^{\infty} Q_{k} x^{2 k}\right)\left(-2 \sum_{n=0}^{\infty} \phi(2 n) x^{2 n}\right),
$$

and the asserted formula follows.

Proposition 3. Suppose $k, l, m \in \mathbb{N} \cup\{0\}$ with $2 k+l \geq 1$. Then

$$
\begin{aligned}
& A(2 m+1 ; 2 k+1, l) \\
& =2 \sum_{\nu=0}^{m} \phi(2 m-2 \nu) \sum_{j=0}^{k} \phi(2 k-2 j) \\
& \quad \times \sum_{\mu=0}^{j} \frac{(i \theta)^{2 \mu}}{(2 \mu+1) !}\left(\begin{array}{c}
2 \nu+1+2 j-2 \mu \\
2 j-2 \mu
\end{array}\right) \zeta(2 \nu+2+2 j+l-2 \mu) .
\end{aligned}
$$

Proof. Corollary 2 with $\theta=\pi$ and $d=2 q+3$, where $q \geq 0$, gives

$$
\begin{aligned}
\sum_{j=0}^{k} \phi(2 k-2 j) \sum_{\mu=0}^{j} \frac{(i \pi)^{2 \mu}}{(2 \mu+1) !}\left(\begin{array}{c}
2 q+1+2 j-2 \mu \\
2 j-2 \mu
\end{array}\right) \zeta(2 q+2+2 j+l-\mu) \\
=-\sum_{\nu=0}^{q} A(2 q+1-2 \nu ; 2 k+1, l) \frac{(i \pi)^{2 \nu}}{(2 \nu+1) !} .
\end{aligned}
$$

Then, by applying Lemma 8 with $P_{m}=A(2 m+1 ; 2 k+1, l)$, and

$$
\begin{aligned}
Q_{m}= & -\sum_{j=0}^{k} \phi(2 k-2 j) \sum_{\mu=0}^{j} \frac{(i \pi)^{2 \mu}}{(2 \mu+1) !} \\
& \times\left(\begin{array}{c}
2 m+1+2 j-2 \mu \\
2 j-2 \mu
\end{array}\right) \zeta(2 m+2+2 j+l-2 \mu),
\end{aligned}
$$

for $m \geq 0$, we obtained the asserted formula for $A(2 m+1 ; 2 k+1, l)$.

By applying Proposition 3 in the case $m=k$ and $l=2 k+1$ for $k \in$ $\mathbb{N} \cup\{0\}$, and using (2.14) and (3.8), we have the following formula. This gives a partial answer to the Subbarao-Sitaramachandrarao problem posed in $[4, \S 5]$. 
Corollary 3. For $k \in \mathbb{N} \cup\{0\}$,

$$
\begin{aligned}
\sum_{m, n=1}^{\infty} \frac{(-1)^{m+n}}{m^{2 k+1} n^{2 k+1}(m+n)^{2 k+1}} & \\
= & -2 \sum_{j=0}^{k}\left(\begin{array}{c}
2 k+2 j+1 \\
2 j+1
\end{array}\right) \phi(2 k-2 j) \phi(4 k+2 j+3) \\
& +4 \sum_{\nu=0}^{k} \phi(2 k-2 \nu) \sum_{j=0}^{k} \phi(2 k-2 j) \sum_{\mu=0}^{j}\left(\begin{array}{c}
2 \nu+1+2 j-2 \mu \\
2 j-2 \mu
\end{array}\right) \\
& \times \zeta(2 k+2 \nu+2 j-2 \mu+3) \frac{(i \pi)^{2 \mu}}{(2 \mu+1) !} .
\end{aligned}
$$

REMARK. In the same way as above, it follows from Corollary 2 that

$$
\begin{aligned}
A(2 m ; 2 k, j)= & -2 \sum_{j=1}^{m} \phi(2 m-2 j) \sum_{\nu=0}^{k} \phi(2 k-2 \nu) \\
& \times \sum_{\mu=0}^{\nu-1}\left(\begin{array}{c}
2 j+2 \nu-2 \mu-1 \\
2 \nu-2 \mu-1
\end{array}\right) \zeta(2 j+2 \nu+l-2 \mu) \frac{(i \pi)^{2 \mu}}{(2 \mu+1) !} \\
& +\zeta(2 k+l) \phi(2 m),
\end{aligned}
$$

when $2 k+l \geq 2$ and $m \geq 1$. In particular, it follows from (2.13) that we can also evaluate

$$
\sum_{m, n=1}^{\infty} \frac{(-1)^{m+n}}{m^{2 k} n^{2 k}(m+n)^{2 k}}+2 \sum_{m, n=1}^{\infty} \frac{(-1)^{n}}{m^{2 k} n^{2 k}(m+n)^{2 k}}
$$

for $k \in \mathbb{N}$; however, neither of the two sums in (4.2) can be deduced separately.

By substituting (4.1) into (3.12) in the case $l=2 p$ and $d=2 q+1$, we obtain

Theorem 2. For $k, p \in \mathbb{N} \cup\{0\}$ with $k+p \geq 1$ and $q \in \mathbb{N}$,

$$
\begin{aligned}
T(2 k+1,2 p, 2 q+1)-T(2 k+1,2 q+1,2 p)+T(2 p, 2 q+1,2 k+1) \\
=-2 \sum_{j=0}^{k}\left(2^{1-2 k+2 j}-1\right) \zeta(2 k-2 j) \\
\quad \times \sum_{\nu=0}^{j}\left(\begin{array}{c}
2 q+1+2 j-2 \nu \\
2 j+1-2 \nu
\end{array}\right) \zeta(2 q+2+2 j+2 p-2 \nu) \frac{(i \pi)^{2 \nu}}{(2 \nu) !}
\end{aligned}
$$




$$
\begin{aligned}
& +4 \sum_{N=0}^{q}\left\{\sum_{\nu=0}^{q-N}\left(2^{1-2 q+2 N+2 \nu}-1\right) \zeta(2 q-2 N-2 \nu)\right. \\
& \times \sum_{j=0}^{k}\left(2^{1-2 k+2 j}-1\right) \zeta(2 k-2 j) \\
& \left.\times \sum_{\mu=0}^{j}\left(\begin{array}{c}
2 \nu+1+2 j-2 \mu \\
2 j-2 \mu
\end{array}\right) \zeta(2 \nu+2+2 j+2 p-2 \mu) \frac{(i \pi)^{2 \mu}}{(2 \mu+1) !}\right\} \frac{(i \pi)^{2 N}}{(2 N) !} .
\end{aligned}
$$

EXAMPLE. We list several nontrivial relations for $T(r, s, N-r-s)$ deduced from Theorem 2 when $N=10,12$ :

$$
\begin{aligned}
& T(3,2,5)-T(3,5,2)+T(2,5,3)=-\frac{1}{935550} \pi^{10} \\
& T(3,2,7)-T(3,7,2)+T(2,7,3)=-\frac{17}{127702575} \pi^{12} \\
& 2 T(5,2,5)-T(5,5,2)=-\frac{19}{91216125} \pi^{12} \\
& T(5,4,3)-T(5,3,4)+T(4,3,5)=\frac{19}{182432250} \pi^{12}
\end{aligned}
$$

\section{References}

[1] K. Dilcher, Zeros of Bernoulli, generalized Bernoulli and Euler polynomials, Mem. Amer. Math. Soc. 386 (1988).

[2] J. G. Huard, K. S. Williams and N. Y. Zhang, On Tornheim's double series, Acta Arith. 75 (1996), 105-117.

[3] L. J. Mordell, On the evaluation of some multiple series, J. London Math. Soc. 33 (1958), 368-371.

[4] M. V. Subbarao and R. Sitaramachandrarao, On some infinite series of L. J. Mordell and their analogues, Pacific J. Math. 119 (1985), 245-255.

[5] L. Tornheim, Harmonic double series, Amer. J. Math. 72 (1950), 303-314.

Department of Management Informatics

Tokyo Metropolitan College

Akishima, Tokyo 196-8540, Japan

E-mail: tsumura@tmca.ac.jp 